\title{
A Novel Ternary Content Addressable Memory (TCAM) Design Using Reversible Logic
}

\author{
1. E.SPURTHI SOWJANYA ,2. N.VINOD KUMAR,Assistant Professor \\ 1,2.Department of ECE,Dr.K.V.Subba Reddy College of Engineering For Women \\ 1.eladisowjanya20@gmail.com,2.vinod.nayakallu@gmail.com
}

\begin{abstract}
Reversible computing is a model of computing where the computational process to some extent is reversible. A necessary condition for reversibility of a computational model is that the relation of the mapping states of transition functions to their successors should at all times be one-to-one. Content addressable memory is a special type of memory which can do search operation in a single clock cycle. CAM has disadvantages of high power dissipation during the matching operation. Ternary content addressable memory (TCAM) is a special type of memory which is used to search for logic 0, logic 1, logic 'x'. These types of memory are used in routers in order to perform the lookup table function in a single clock cycle. As the use of networks, typified by the Internet, has spread widely in recent years, attention has focused on TCAMs as a key device for increasing the speed of packet forwarding (packet data transfers) by networking equipment by enabling high-speed lookup of destinations, etc., for large volumes of information during packet data transfers. Reversible logic has gained its interest in recent years due to its ultra low power characteristics. Many works have been done to reduce the power consumption in TCAM. This paper deals with a novel design of TCAM cells using reversible logic. The proposed design is optimized in terms of number of garbage outputs and quantum cost. The proposed TCAM cell does the function of the conventional TCAM cell.
\end{abstract}

Keywords-TCAM, Garbage output, Quantum cost, Reversible logic, Content addressable memory, Power consumption

I.INTRODUCTION

Ternary content addressable memory compares input data against stored data (logic ' 0 ', logic ' 1 ', don't care) in parallel and outputs the matched data. TCAM find its applications in routers and some networking equipment due to its deterministic and high speed searches by using parallel operation to compare the data stored with the search words. RAM needs an address to fetch the data stored in the memory whereas CAM need the contents to be searched in the search lines and the matched address is obtained at the output. CAM is known for its high speed search operation. However, the CAMs consume lot of power due to its high speed operation and lot of heat is dissipated. High power consumption in CAM devices increases the junction temperatures of the chip which increases the heat dissipation there by reducing the performance of the chip. When there is computation in any logic circuits, the loss in information will lead to heat generation in the system. Landauer proposed that for every single bit loss of information there will be KTln2 Joules of energy will be dissipated in the form of heat. Bennett showed that heat dissipation will be zero ideally if the circuit is designed using reversible elements. According to the definition of reversible elements, the circuit is said to be reversible if there is no loss in information. Feynman has proposed the first $2 \times 2$ reversible gate which will do ex or operation. Now-a-days, the research in designing the irreversible circuits to reversible circuits is the great area of concern due to its low power characteristics of the design. In the conventional TCAM design, 16 transistors are used to do the search and match operations. The change in state of the transistors due to the changes in the bit of searchline and match-line will lead to the heat dissipation of the circuits. It has been shown that the power consumption for the conventional 16T TCAM cell is approximately $12 \mathrm{~mW}$ to $15 \mathrm{~mW}$ of power. This paper aims at presenting a novel TCAM design using reversible elements as an alternate to reduce the power consumption in the TCAM cell. A novel SRAM cell is designed using the reversible elements which are used to store the data. The match line and the search line are also designed using reversible elements which emulate the conventional NAND type TCAM cell. To the best of our knowledge, this is the first paper on designing a novel TCAM cell using reversible elements. The design is verified by using Xilinx ISE simulator.

The rest of the paper is organized as follows. Section II discusses about the conventional TCAM cell. Section III discusses about the reversible elements and the TCAM cell. Section IV discusses about the SRAM cell using reversible elements in brief. Section V discusses about TCAM cell design using reversible elements. Section VI discusses about Reversible Encoder and Decoder. Section VII illustrates the search operation in TCAM array. Section VIII discusses about the results of the proposed design. Section IX concludes the paper.

\section{II.LITERATURE REVIEW}

Content addressable memory is of two types based on the types of bits stored in it. Each and every CAM cells will have their own comparison and search circuitry in order to search and gives the address of the matched data. Binary CAM can store logic ' 0 ' and logic ' 1 ' whereas ternary CAM can store logic ' 0 ', logic ' 1 ' and don't care. Thus in TCAM, the stored key $11 \mathrm{x}$ can match any one of the search keys of 110 or 
111. TCAM cells are arranged in the 2D array format.

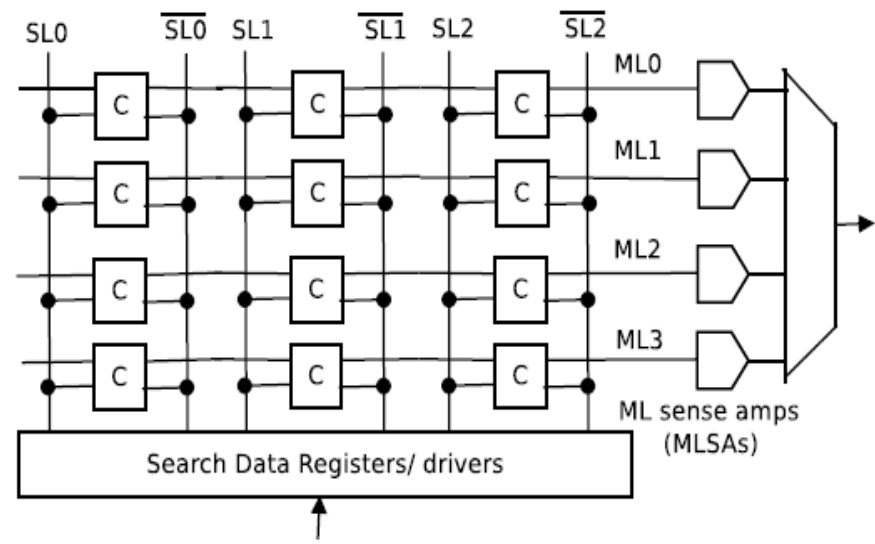

Fig. 1: Conventional $4 \times 3$ TCAM Array

The row cells will have common match line which is used to enable or disable the whole row based on matching condition or mismatching condition. The column cells will have common search line which is used to search the whole column. A conventional TCAM array is shown in Figure 1.

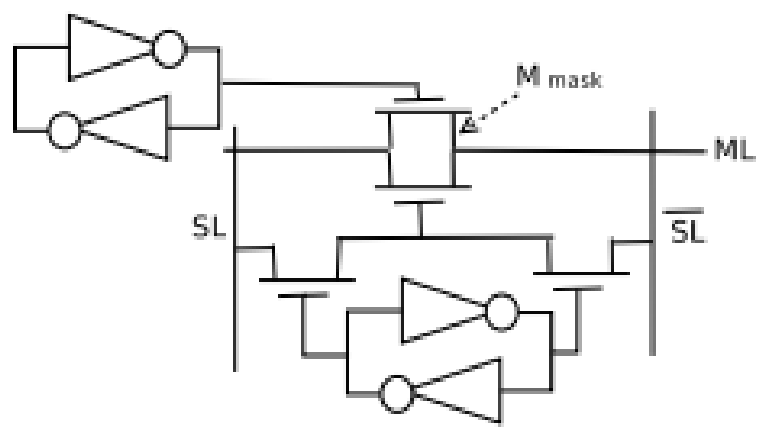

Fig. 2: Conventional NAND type TCAM Cell

Figure 1 shows the conventional $4 \times 3$ TCAM array with 4 rows (TCAM words) and 3 columns. The cells in the same row are connected through the common match-line and the cells in the column have common search-line. There are 3 stages in the conventional TCAM cell. Search line (SL) precharge, matchline (ML) precharge, match-line (ML) evaluate. During the search-line precharge operation, the search-line will be made high in order to disconnect the TCAM cells from the pull down path. In the next stage, that is during match-line precharge, the match-line will be charged high and in the last stage of the operation the match-line will be evaluate by placing the search bits in the search lines. If the data are matched then the match-line will be in high state else the match line state will discharge through the pull down path which proves the irreversibility operation of the TCAM cells. Figure 2 shows the conventional NAND type TCAM cell.

Figure 3 shows the usage of TCAM in routing table. The search data are given through the search registers. The address look up matches both entry 2 and entry 3. By using longest prefix matching method entry 2 will be selected and its address is passed to the priority encoder. This will be decoded and the corresponding address will be matched by the RAM.
One approach that is well defined in the literature for practical implementation of reversible circuits is to never turn a switch ON or OFF when there is either voltage across it or current going through it. Reversible energy recovery logic uses this approach in order to avoid the non-adiabatic losses, which is essential for an ultra low power energy system. As a proof of concept for low power energy consumption using reversible logic, an ultra low power 16-bit carry look ahead adder was proposed in using reversible energy recovery logic.

CAM

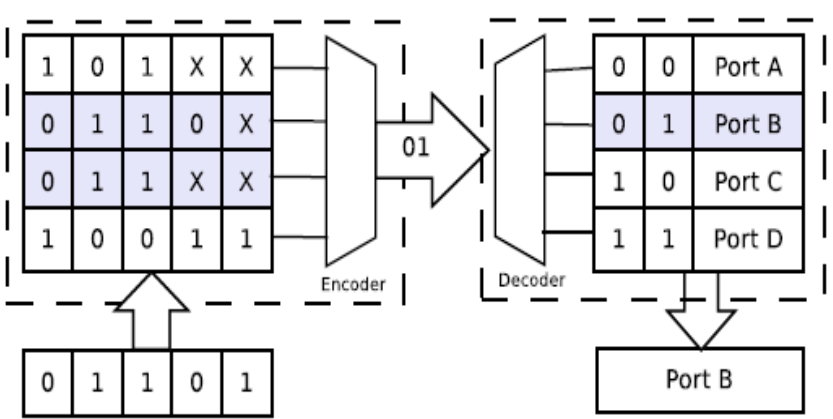

Fig. 3: Application of TCAM in Routing Table

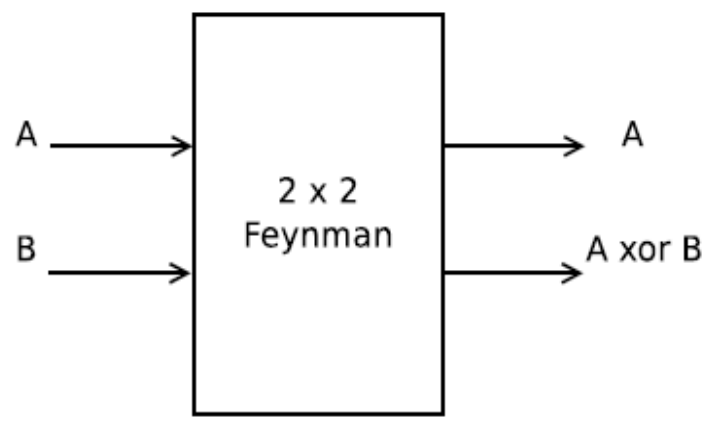

Fig. 4: Feynman Gate

\section{III.REVERSIBLE ELEMENTS}

This section discusses about the basics of reversible elements. According to the definition of the reversible circuit, there should not be any loss in the information. The design constraints in the reversible circuit is that the input and output vectors must map uniquely. There should be equal number of inputs and outputs and fan outs are not allowed in the design. Feedback from the output to the input of the same gate is not allowed. The design parameters in the reversible element design are quantum cost, worst case delay and the garbage output. Quantum cost is defined as the number of $1 \times 1$ reversible gate or $2 \times 2$ reversible gates. Worst case delay is defined as the number of delay units to produce the output for the given input. The worst case delay of the $1 \times 1$ reversible gate is defined as 1 unit of delay. Garbage output is the number of unused output to ensure the reversibility property of the circuits designed. Feynman has proposed the $2 \times 2$ Feynman gate which performs the operation of the ex or gate. Figure 4 shows the $2 \times 2$ Feynman gate with inputs and outputs. 
Figure 5 shows the $3 \times 3$ Fredkin gate with inputs and outputs. As per the definition of quantum cost, the quantum cost of $2 \times 2$ Feynman gate is 1 and $3 \times 3$ Fredkin gate is 5 . Feynman gate can be used as the fanout gate with one input as 0 . Feynman gate can also act as not gate with the B input as 1 which will invert the value of $\mathrm{A}$.

Fredkin gate can act as multiplexer with the input $\mathrm{A}$ as the selection line. Peres has introduced $3 \times 3$ Peres gate. Figure 6 shows the Peres gate. Peres gate can be used as logical NAND or AND gate based on the values of input C.

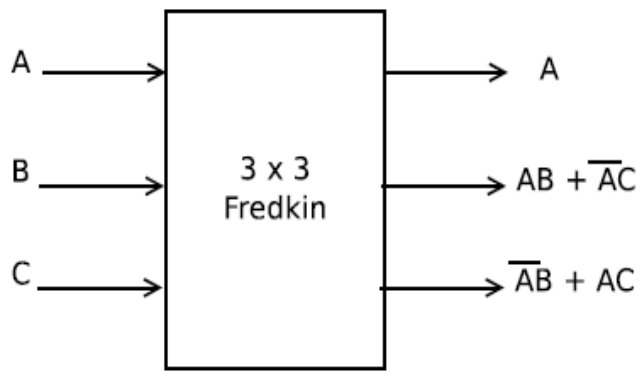

Fig. 5: Fredkin Gate

The quantum cost of the Peres gate is 4 and the worst case delay is 4 . Reversible element based SRAM cell was designed in [9]. The main disadvantage in the design [9] is that quantum cost of the proposed SRAM cell can be further reduced and the reversible decoder proposed does not contain enable signal which is essential for chip select.

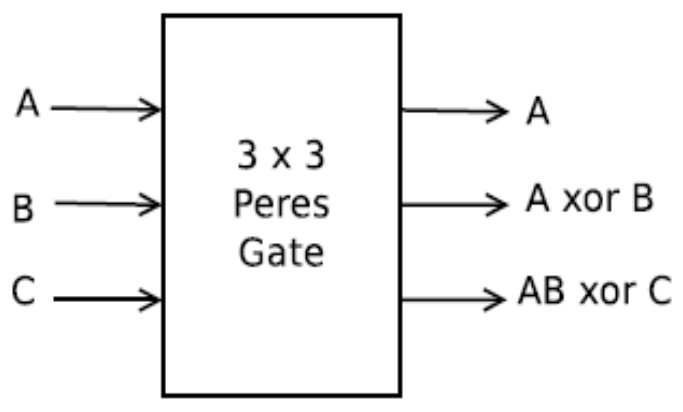

Fig. 6: Peres Gate

\section{IV.SRAM CELL BASED ON REVERSIBLE ELEMENTS}

This section discusses about the novel SRAM cell design based on reversible elements. Figure 7 shows the proposed SRAM cell using reversible gates. In this design, $3 \times 3$ Fredkin gate and the $2 \times 2$ Feynman gate is used to form the SRAM cell which is used to store the single bit of information. Each and every SRAM cell will have word line (W L) in order to make the SRAM cell to function in one of the modes that is either in read/write or hold state. If the value of the $\mathrm{W} L=0$ then the previous value has to be hold by the SRAM cell. If the value of $\mathrm{W} L=1$, then the SRAM will be in any one of the read/write state based on the external write/sense circuits.

The WL output in the SRAM cell is used to enable the row cells which emulate the functionality of the conventional SRAM cell. Quantum cost for the proposed SRAM cell is 6 . Figure 8 shows the SRAM cell with $\mathrm{read} / \mathrm{write}$ enable with the row decoder. The quantum cost of this design is 16 which is still less as compared to the SRAM cell proposed in [9]. This design is used to store the bit in the TCAM memory cells.

The design proposed in Figure 8 emulates the functionality of SRAM array with the reversible read circuits, reversible write circuits. The row decoder in [10] is used in the designing of SRAM array.

The quantum cost of the decoder proposed in [9] is 10 whereas the quantum cost of the decoder proposed in [10] is 15 . But the decoder proposed in [10] is with the enable bit which is very essential in the design of memories for the chip select.

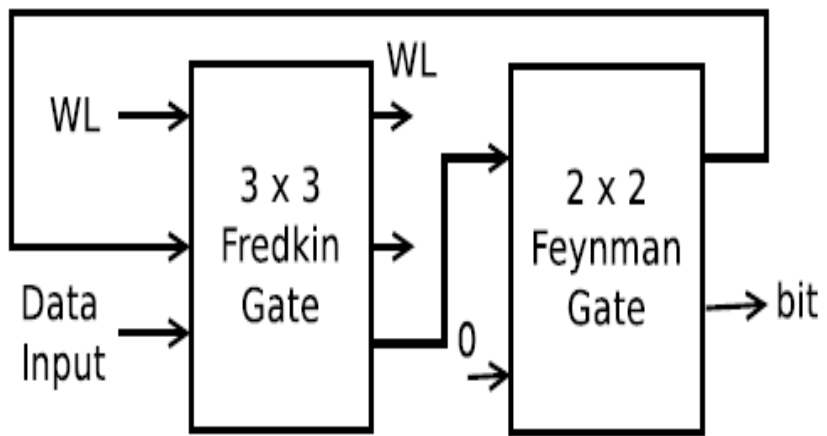

Fig. 7: SRAM Cell using Reversible Gates

V.PROPOSED TCAM CELL USING REVERSIBLE ELEMENTS

This section discusses about the proposed TCAM cell using reversible elements. Figure 9 shows the proposed TCAM cell. SRAM cell discussed in the previous section is used to store the bits. The data will be stored initially in the SRAM cell using the reversible SRAM cell as discussed in the previous section. The data stored will be passed to the $2 \times 2$ Feynman gate with the search line as one input. So, the search line will be duplicated and ex-or operation of data and the search-line data will be the other output. If both search line data and the data stored are same, then the output of the Feynman gate is search-line data and zero. The search line data will be passed to the next TCAM cell of the same column. The output of ex-or operated value is passed to the next $2 \times 2$ Feynman gate which will act like a not gate with one of the input as constant input 1 . So, if the ex-or operated value is 0 , then the output of second Feynman is logic ' 1 '. The value obtained in the second $2 \times 2$ Feynman gate is passed to the $3 \times 3$ Fredkin gate. Inputs to the $3 \times 3$ Fredkin gate are match bit $(\mathrm{M})$, output from $2 \times 2$ Feynman gate and the constant value of logic ' 1 '. Table I illustrates the functionality of the TCAM cell using the match bit. 
TABLE I: Truth Table for Proposed Reversible TCAM Cell

\begin{tabular}{|c|c|c|c|}
\hline Data Stored & Search Bit & Match Bit & Match-line State \\
\hline 0 & 0 & 0 & 1 \\
\hline 0 & 0 & 1 & 1 \\
\hline 0 & 1 & 0 & 0 \\
\hline 0 & 1 & 1 & 1 \\
\hline 1 & 0 & 0 & 0 \\
\hline 1 & 0 & 1 & 1 \\
\hline 1 & 1 & 0 & 1 \\
\hline 1 & 1 & 1 & 1 \\
\hline
\end{tabular}

If the match bit value is logic ' 1 ', then it is a don't care term which should match in all conditions. If the value of match bit (M) is logic ' 0 ', then it is bit value. Based on the search line and bit line matching the match line is made to be logic high. The values from the Fredkin gate is passed to the 3 $\times 3$ Peres gate which performs the function of the match-line. In Peres gate $\mathrm{C}$ input is made as 0 . So, the output of the Peres gate will be logic 'AND' operation of the previously matched line in the row with the match line of the current TCAM cell. So, if the previous values of the matched line are zero, then the match line for the whole row will be maintained low. If the matchline in all the TCAM cells is high, then the match-line will be at high. If at least any one of the match-line of the TCAM cell is low then the match-line of the whole row will be at low which resembles the functionality of the conventional TCAM cell.

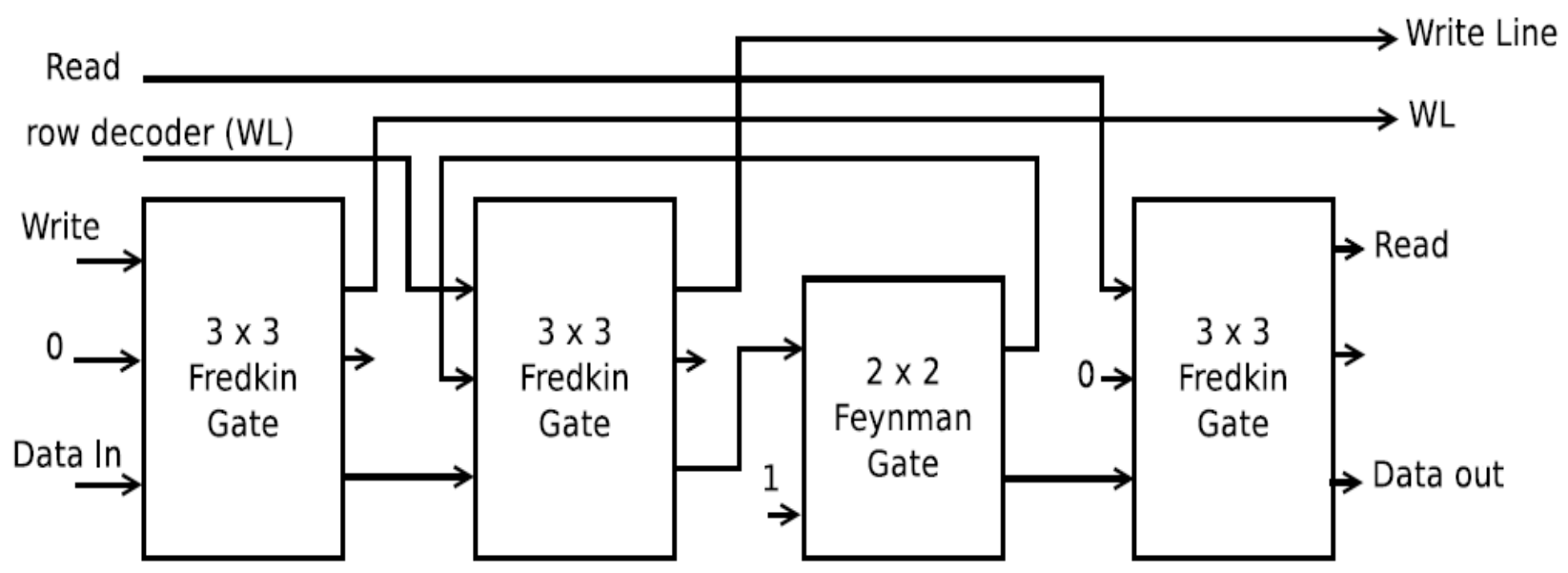

Fig. 8: Reversible SRAM Cell with Read and Write Signals

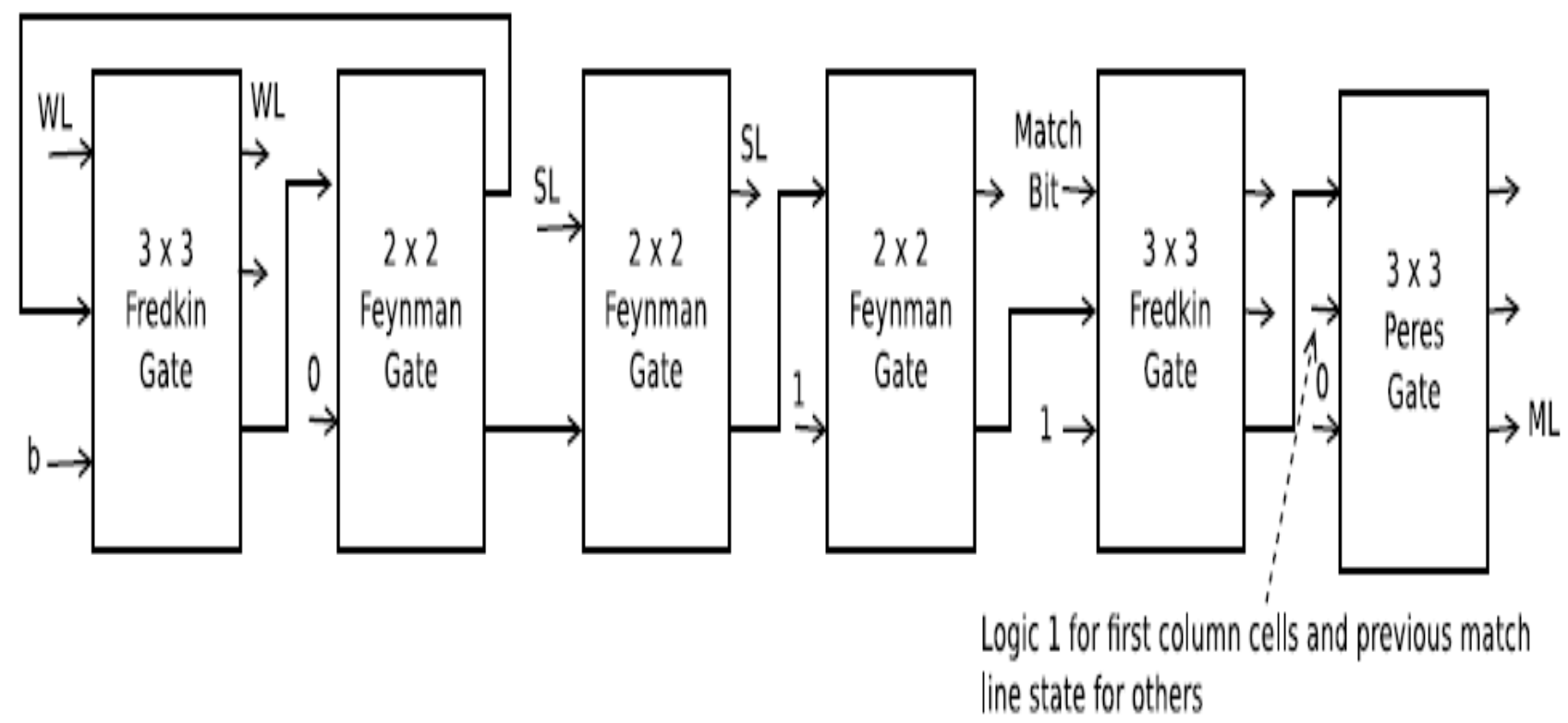

Fig. 9: Reversible TCAM Cell 


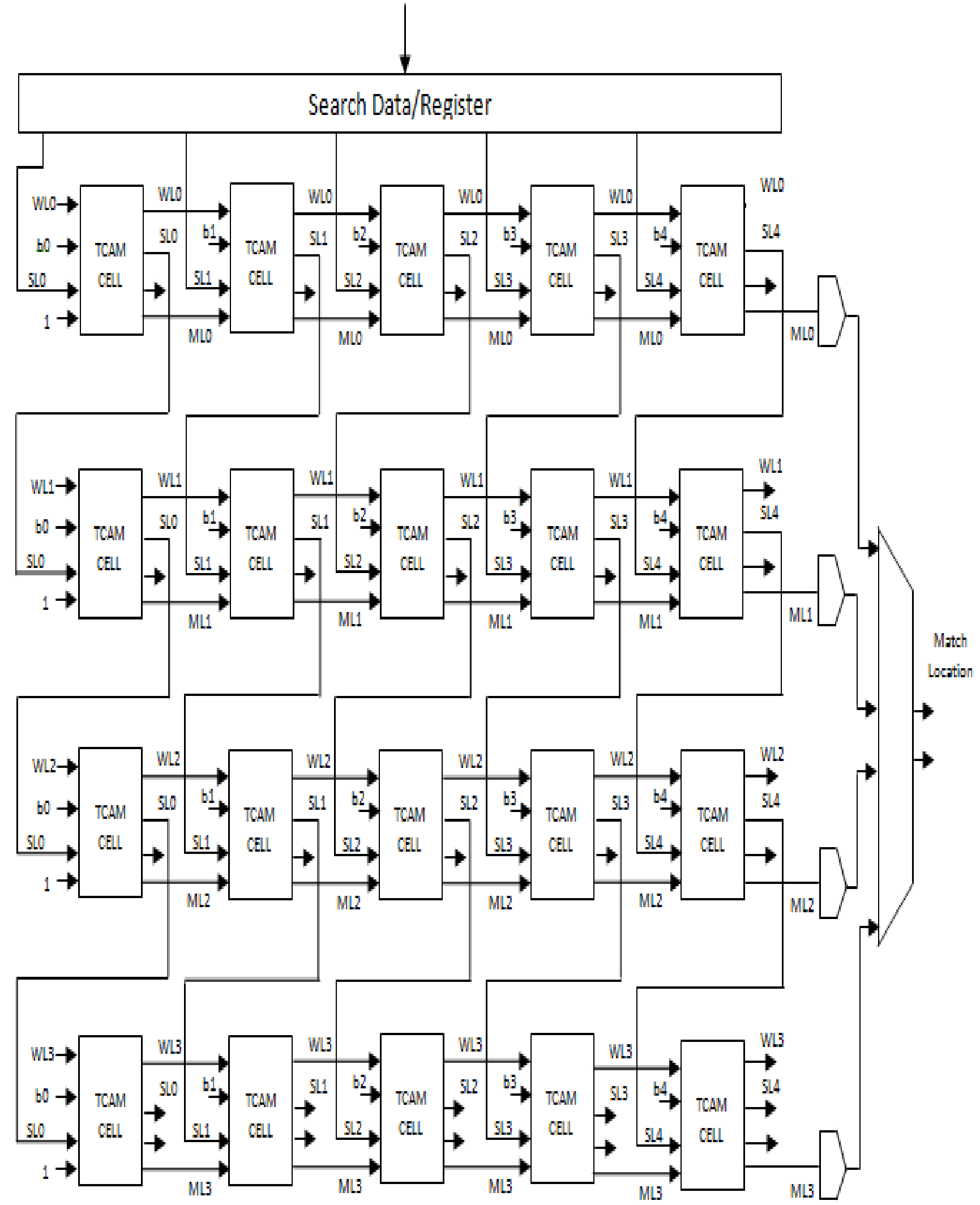

Fig. 10: Reversible 4 x 5 TCAM Array 


\section{REVERSIBLE ENCODER AND DECODER}

The proposed 4:2 Reversible Encoder is shown in figure 11. It uses three Feynman gates (FG) and one Fredkin gate (FRG).It has four inputs $\mathrm{A}, \mathrm{B}, \mathrm{C}, \mathrm{D}$ and two outputs o1\&o 2 and also has two garbage outputs. The operation of circuit is given in Table. This proposed 4:2 Encoder has Quantum cost of 8 .

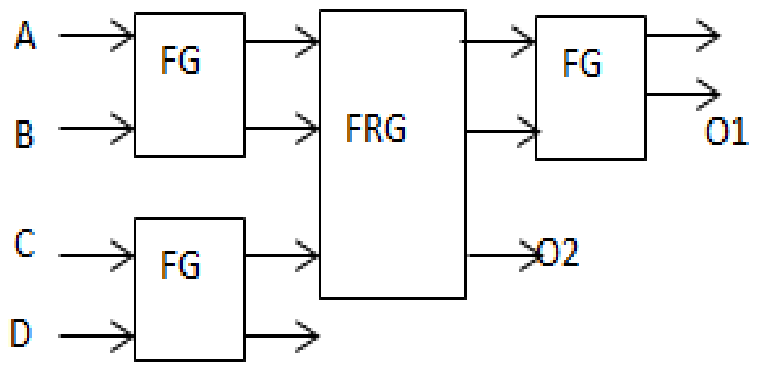

Fig.11: Proposed 4:2 Reversible Encoder.

A single Fredkin gate is capable of working like a 1:2 decoder if the first input is IN1, while the second and third inputs are 0 and 1 respectively.A design of 2:4 decoder using 3 Fredkin gates has been proposed, as shown in Figure 12. The quantum cost of this design is 15 .

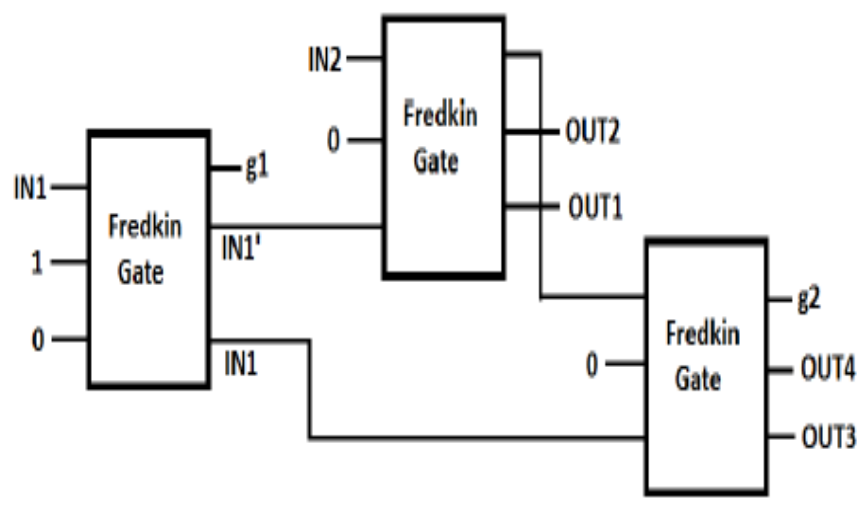

Fig: 12: Proposed reversible 2:4 decoder

\section{SEARCH OPERATION IN TCAM ARRAY}

This section discusses about the operation of TCAM array and how the data are searched and matched. Figure. 10 proposes the $4 \times 5$ reversible TCAM array. The data are stored in each TCAM cell using normal SRAM operation. Once the data is stored in the TCAM cell, match bit will be set either logic ' 0 ' or logic ' 1 ' based on whether the stored value is bit or don't care. Search data are passed to the corresponding TCAM cell from search data registers.

Let us assume the data stored in the first row of the TCAM array be0011X and the data in the search-line be 00101. SL1 data that is 1 is ex-ored with data value that is 1 . So the output of the ex-or operation is 0 . Whenever the data are matched the output of the ex-or operation is 0 which is then inverted to get the output 1 . This resembles the functionality of the conventional binary CAM cell. The data stored in the TCAM is either logic ' 0 '/logic ' 1 ' or don't care bit based on the value of the match bit.
Quantum Cost (QC), Gate Count (GC) and Constant Inputs (CI).

\begin{tabular}{|c|c|c|c|c|}
\hline Reversible Design & GO & QC & GC & CI \\
\hline SRAM & 2 & 9 & 2 & 1 \\
\hline SRAM(R/W) & 3 & 16 & 4 & 3 \\
\hline TCAM & 6 & 17 & 6 & 4 \\
\hline ENCODER & 2 & 8 & 4 & 0 \\
\hline DECODER & 2 & 15 & 3 & 4 \\
\hline
\end{tabular}

If the value of the match bit is ' 0 ' then the data will be either logic ' 0 ' or logic ' 1 '. If the match bit is ' 1 ' then the data stored is don't care. If $M=1$, then the output is logic ' 1 ' at all cases of input. If the value of $M=0$ then the output will be logic ' 0 ' or logic ' 1 ' based on the matching of search data and the data stored. The output from the $3 \times 3$ Fredkin gate is passed to the $3 \times 3$ Peres gate. The inputs to the Peres gate are the output from the $3 \times 3$ Fredkin gate, previous matchline values and the constant input ' 0 '. The previous match-line value of the first TCAM cell is set to be 1 which resembles the match line precharge in the conventional TCAM cell. The match-line output of the first TCAM cell is passed to the $3 \times 3$ Peres gate of the second TCAM cell.

If the match-line of the first TCAM cell is zero then the whole match-line has to be pull down to zero. So, the match-line which is zero is 'and' operated with the second match-line which will result in the match line low. This shows the operation of reversible TCAM array which performs the same operation of the conventional TCAM array. The match-line is passed through the whole row of the cell and the search line is passed to the whole column of the TCAM cell.

\section{SIMULATION RESULTS}

All the synthesis and simulation results are performed using Verilog HDL. The synthesis and simulation are performed on Xilinx ISE 14.4. The simulation results are shown below figures.

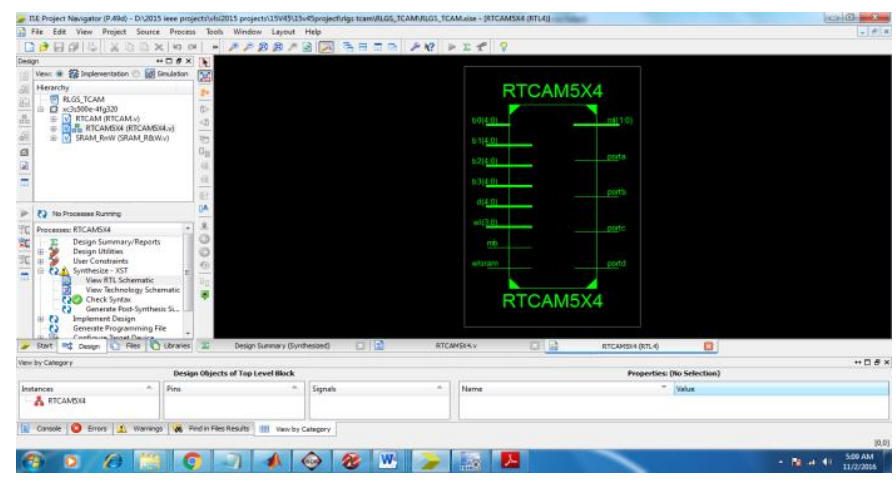

Fig. 13: RTL schematic of Reversible 4X5 TCAM Array

TABL II: Design metrics of Reversible Ternary Contents Address Memory inters of Garbage Outputs (GO), 


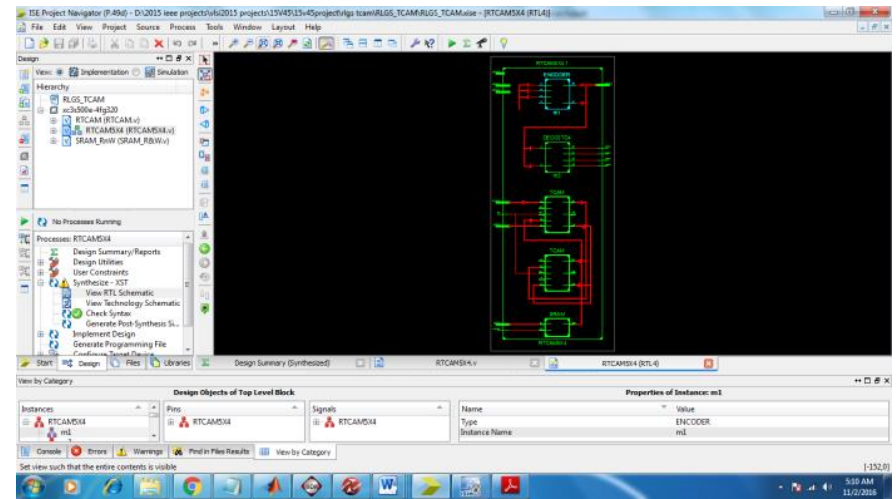

Fig. 14: RTL sub schematic of Reversible 4X5 TCAM Array

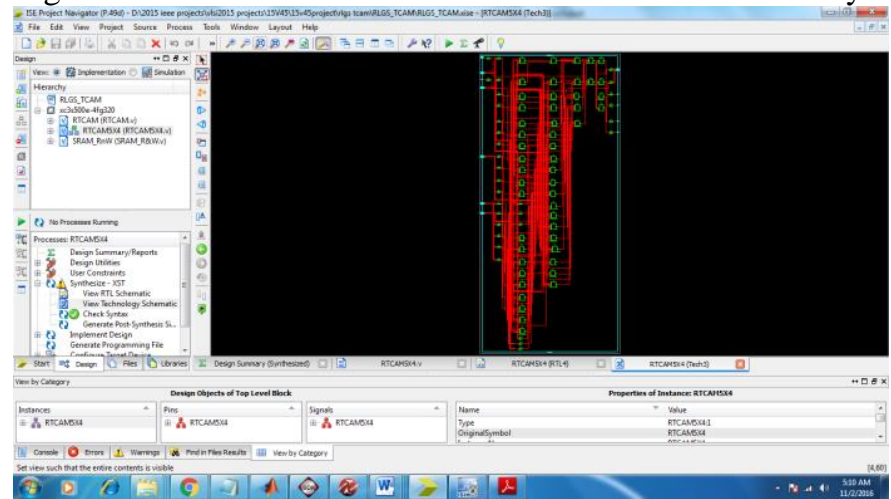

Fig. 15: Technology schematic of Reversible 4X5 TCAM Array

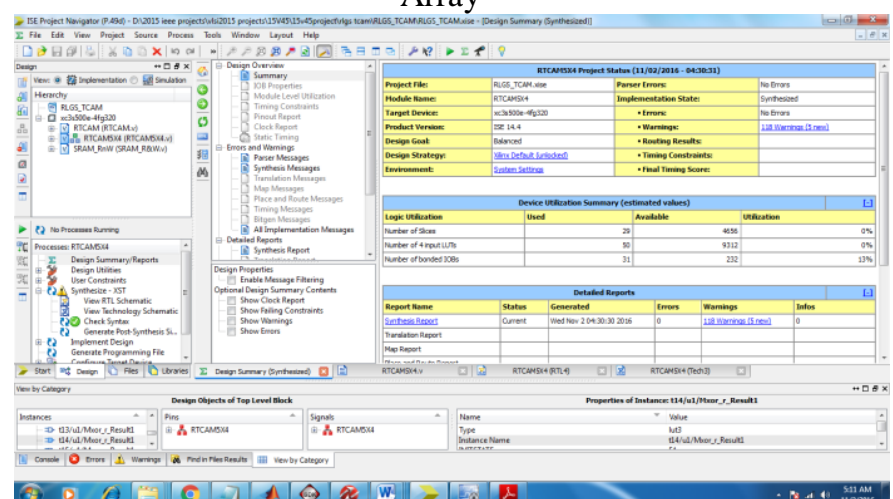

Fig. 16: Design summary of Reversible 4X5 TCAM Array

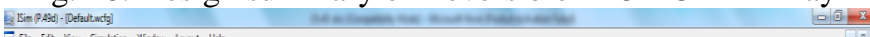

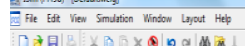

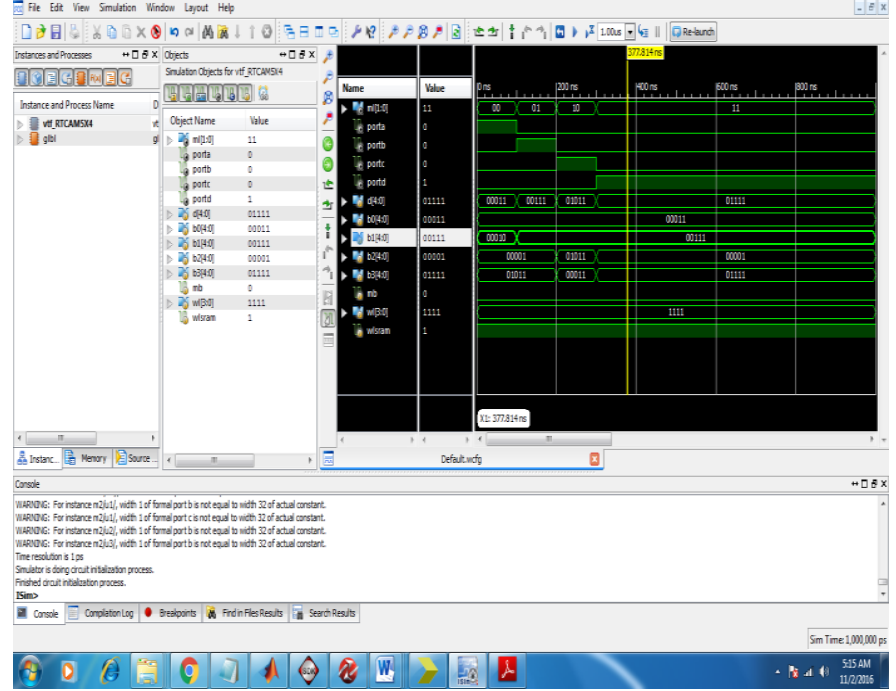

Fig. 17: Simulation of Reversible 4X5 TCAM Array

\section{CONCLUSION AND FUTURE WORK}

This paper proposed a novel design of $4 \times 5$ TCAM array design using reversible circuit design. Ternary content addressable memory compares input data against stored data (logic ' 0 ', logic ' 1 ', don't care) in parallel and outputs the matched data. In reversible SRAM design, $3 \times 3$ Fredkin gate and the $2 \times 2$ Feynman gate is used to form the SRAM cell which is used to store the single bit of information. Each and every SRAM cell will have word line (WL) in order to make the SRAM cell to function in one of the modes that is either in read/write or hold state. $3 \times 3$ Fredkin gate and Peres gate are used to perform the search line and match line operation. The design is verified and simulated by using Xilinx ISE simulator.The practical realization of reversible TCAM based chip will definitely reduce the power consumption of the network switches. In future the proposed Ternary Content Address Memory (TCAM) can be used in networking and routers applications for high speed data searching.

\section{REFERENCES}

[1] N. Mohan, W. Fung, D. Wright, and M. Sachdev, "Design techniques and test methodology for low-power tcams," Very Large Scale Integration (VLSI) Systems, IEEE Transactions on, vol. 14, no. 6, pp. 573-586, 2006.

[2] R. Landauer, "Irreversibility and heat generation in the computing process," IBM Journal of Research and Development, vol. 5, no. 3, pp. 183-191, July 1961.

[3] C. Bennett, "Logical reversibility of computation," IBM Journal of Research and Development, vol. 17, no. 6, pp. 525-532, Nov 1973.

[4] R. Feynman, "Quantum mechanical computers," Foundations of Physics, vol. 16, no. 6, pp. 507-531, 1986.

[5] F. Zane, G. Narlikar, and A. Basu, "Coolcams: Power-efficient tcams for forwarding engines," in INFOCOM 2003. TwentySecond Annual Joint Conference of the IEEE Computer and Communications. IEEE Societies, vol. 1. IEEE, 2003, pp. 42-52.

[6] K. Pagiamtzis and A. Sheikholeslami, "Content-addressable memory (cam) circuits and architectures: a tutorial and survey," Solid-State Circuits, IEEE Journal of, vol. 41, no. 3, pp. 712-727, March 2006.

[7] J. Lim, D.-G.Kim, and S.-I.Chae, "A 16-bit carry-lookahead adder using reversible energy recovery logic for ultra-low-energy systems," Solid-State Circuits, IEEE Journal of, vol. 34, no. 6, pp. 898-903, 1999.

[8] A. Peres, "Reversible logic and quantum computers," Physical review A, vol. 32, no. 6, p. 3266, 1985.

[9] M. Morrison, M. Lewandowski, R. Meana, and N. Ranganathan, "Design of static and dynamic ram arrays using a novel reversible logic gate and decoder," in Nanotechnology (IEEE-NANO), 2011 11th IEEE Conference on, Aug 2011, pp. 417-420.

[10] S. Mahammad and K. Veezhinathan, "Constructing online testable circuits using reversible logic," Instrumentation and Measurement, IEEE Transactions on, vol. 59, no. 1, pp. 101-109, Jan 2010. 